\title{
Four-fermion production near the W-pair production threshold
}

\section{Pietro Falgari*}

Institut für Theoretische Physik E, RWTH-Aachen

E-mail: falgari@physik.rwth-aachen.de

I report on recent results for the total production cross section of the process $e^{-} e^{+} \rightarrow \mu^{-} \bar{v}_{\mu} u \bar{d} X$ near the $W$-pair production threshold up to next-to-leading order in $\Gamma_{W} / M_{W} \sim \alpha \sim v^{2}$ obtained in the framework of unstable-particle effective field theory. Remaining theoretical uncertainties and their impact on the experimental determination of the $W$ mass are discussed.

8th International Symposium on Radiative Corrections

October 1-5, 2007

Florence, Italy

* Speaker. 


\section{Introduction}

An accurate measurement of the $W$ mass is of primary interest for precision tests of the Standard Model and for search of New-Physics effects through virtual-particle exchange. The total error on $M_{W}$ could be lowered to $6 \mathrm{MeV}$ by measuring the four-fermion production cross section near the $W$-pair production threshold [1] at a future International Linear Collider (ILC), provided that the theoretical uncertainties are well below $1 \%$. This is a difficult task, requiring gauge-invariant inclusion of finite-width effects and calculation of QCD and electroweak radiative corrections to the full $2 \rightarrow 4$ process. Previous NLO calculations in the double-pole approximation [2] were supposed to break down near threshold for kinematical reasons. The recent computation of the complete NLO corrections to $e^{-} e^{+} \rightarrow 4 f$ in the complex-mass scheme [3] is valid both near threshold and in the continuum, but is technically difficult, requiring the computation of one-loop six-point functions.

Here I present NLO results for the total cross section of the process

$$
e^{-} e^{+} \rightarrow \mu^{-} \bar{v}_{\mu} u \bar{d} X
$$

near the $W$-pair production threshold [4] computed with effective field theory (EFT) techniques $[5,6,7]$. Section 2 reviews briefly the formalism, while the calculation of the Born cross section and of radiative corrections is outlined in Sections 3 and 4. Section 5 presents numerical results together with an estimate of the remaining theoretical uncertainties and a comparison with [3].

\section{Unstable-particle effective field theory}

The EFT approach [7] exploits the hierarchy of scales $M \Gamma \ll M^{2}$ which characterizes processes involving unstable particles, $M$ and $\Gamma$ being the mass and width of the intermediate resonance. The degrees of freedom of the full theory are classified according to their scaling into short-distance $\left(k^{2} \sim M^{2}\right)$ and long-distance $\left(k^{2} \lesssim M \Gamma\right)$ modes. The fluctuations at the small scale (resonant particles, soft and Coulomb photons,...) represent the field content of the effective Lagrangian $\mathscr{L}_{\text {eff }}$. "Hard" fluctuations with $k^{2} \sim M^{2}$ are not part of the effective theory and are integrated out. Their effect is included in $\mathscr{L}_{\text {eff }}$ through short-distance matching coefficients, computed in standard fixedorder perturbation theory. The systematic inclusion of finite-width effects is relevant for modes with virtuality $k^{2} \lesssim M \Gamma$ and is obtained through complex short-distance coefficients in $\mathscr{L}_{\text {eff }}$ [7].

The specific process (1.1) is primarily mediated by production of a pair of resonant $W \mathrm{~s}$. The total cross section is extracted from appropriate cuts of the forward-scattering amplitude [4], which after integrating out the hard modes with $k^{2} \sim M_{W}^{2}$ reads [7]

$$
i \mathscr{A}=\sum_{k, l} \int d^{4} x\left\langle e^{-} e^{+}\left|\mathrm{T}\left[i \mathscr{O}_{p}^{(k) \dagger}(0) i \mathscr{O}_{p}^{(l)}(x)\right]\right| e^{-} e^{+}\right\rangle+\sum_{k}\left\langle e^{-} e^{+}\left|i \mathscr{O}_{4 e}^{(k)}(0)\right| e^{-} e^{+}\right\rangle .
$$

The operators $\mathscr{O}_{p}^{(l)}\left(\mathscr{O}_{p}^{(k) \dagger}\right)$ in the first term on the right-hand side of (2.1) produce (destroy) a pair of non-relativistic resonant $W$ bosons. The second term accounts for the remaining non-resonant contributions. The computation of $\mathscr{A}$ is split into the determination of the matching coefficients of the operators $\mathscr{O}_{p}^{(l)}, \mathscr{O}_{4 e}^{(k)}$ and the calculation of the matrix elements in (2.1). Both quantities are computed as power series in the couplings $\alpha, \alpha_{s}$, the ratio $\Gamma_{W} / M_{W}$ and the non-relativistic velocity of the intermediate resonant $W$ pair $v^{2} \equiv\left(\sqrt{s}-2 M_{W}\right) /\left(2 M_{W}\right)$, collectively referred to as $\delta \sim \alpha_{s}^{2} \sim \alpha \sim \Gamma_{W} / M_{W} \sim v^{2}$. 
The effective Lagrangian describing the non-relativistic $W$ bosons up to NLO in $\delta$ is [6]

$$
\mathscr{L}_{\mathrm{NRQED}}=\sum_{a=\mp}\left[\Omega_{a}^{\dagger i}\left(i D^{0}+\frac{\vec{D}^{2}}{2 M_{W}}-\frac{\Delta}{2}\right) \Omega_{a}^{i}+\Omega_{a}^{\dagger i} \frac{\left(\vec{D}^{2}-M_{W} \Delta\right)^{2}}{8 M_{W}^{3}} \Omega_{a}^{i}\right] .
$$

$\Delta$ is the matching coefficient $\Delta \equiv\left(\bar{s}-M_{W}^{2}\right) / M_{W}$, where $\bar{s}$ is the complex pole of the $W$ propagator. The field $\Omega_{ \pm}^{i}=\sqrt{2 M_{W}} W_{ \pm}^{i}$ describes the three physical polarizations of non-relativistic $W \mathrm{~s}$, and the covariant derivative $D_{\mu} \Omega_{ \pm}=\left(\partial_{\mu} \mp i e A_{\mu}\right) \Omega_{ \pm}$contains the interaction of the resonant fields $\Omega_{ \pm}$with soft and potential photons (see Section 4). To complete $\mathscr{L}_{\text {eff }}$ one has to add to (2.2) the effective production vertices $\mathscr{O}_{p}^{(l)}$ and the four-fermion operators $\mathscr{O}_{4 e}^{(k)}$ with the corresponding matching coefficients computed to the desired order in $\delta$. These are presented in Sections 3 and 4.

\section{EFT approximation to the Born cross section}

The lowest-order production operator of two non-relativistic resonant $W \mathrm{~s}$ is [6]

$$
\mathscr{O}_{p}^{(0)}=\frac{\pi \alpha_{e w}}{M_{W}^{2}}\left(\bar{e}_{c_{2}, L}\left(\gamma^{i} n^{j}+\gamma^{j} n^{i}\right) e_{c_{1}, L}\right)\left(\Omega_{-}^{\dagger i} \Omega_{+}^{\dagger j}\right) .
$$

Its matching coefficient is extracted from the on-shell process $e^{-} e^{+} \rightarrow W^{-} W^{+}$, where "on-shell" means $k^{2}=\bar{s}$. The four-fermion operators $\mathscr{O}_{4 e}^{(k)}$ do not contribute to $\mathscr{A}$ at this order, and the forward-scattering amplitude is simply

$$
i \mathscr{A}^{(0)}=\int d^{4} x\left\langle e^{-} e^{+}\left|T\left[i \mathscr{O}_{p}^{(0) \dagger}(0) i \mathscr{O}_{p}^{(0)}(x)\right]\right| e^{-} e^{+}\right\rangle=\underbrace{}_{\Omega} \underbrace{\Omega}_{p} \mathcal{O}_{p}^{(0)} e_{e}^{e}=-\frac{i \pi \alpha^{2}}{s_{w}^{4}} \sqrt{-\frac{E+i \Gamma_{W}^{(0)}}{M_{W}}}
$$

with $E=\sqrt{s}-2 M_{W}$ and $s_{w}=\sin \theta_{W}$. The total cross section for (1.1) is extracted from appropriate cuts of (3.2). At lowest order this is correctly done by multiplying the imaginary part of $\mathscr{A}^{(0)}$ with the LO branching ratios of the decays $W^{-} \rightarrow \mu^{-} \bar{v}_{\mu}, W^{+} \rightarrow u \bar{d}$, so that $\sigma^{(0)}=\frac{1}{27 s} \operatorname{Im} \mathscr{A}^{(0)}$.

Beyond the leading term $\sigma^{(0)}$ there are contributions which can be identified with terms of the expansion in $\delta$ of a full-theory Born result computed with a fixed-width prescription. The first class of corrections arises from four-electron operators in (2.1). The imaginary part of their matching coefficients are extracted from suitable cuts of hard two-loop SM diagrams [4]:

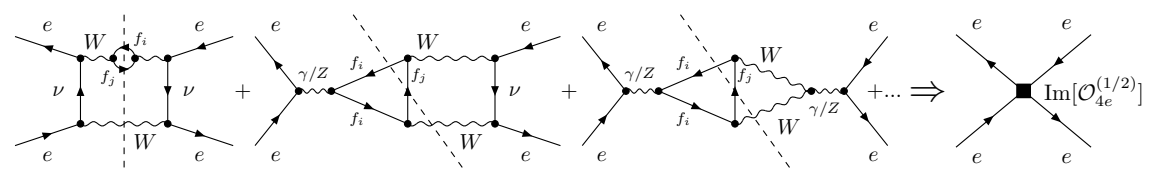

Compared to the LO cross section $\sigma^{(0)} \sim \alpha^{2} \sqrt{\delta}$ the new term is suppressed by $\alpha / \sqrt{\delta} \sim \sqrt{\delta}$ and is denoted as " $\sqrt{\mathrm{N}} L \mathrm{O}$ ". True NLO contributions to $\mathscr{A}^{(0)}$ arise from higher-dimension production operators and propagator corrections. The former come from the matching of the effective theory on the on-shell process $e^{-} e^{+} \rightarrow W^{-} W^{+}$at order $v\left(\mathscr{O}_{p}^{(1 / 2)}\right)$ and $v^{2}\left(\mathscr{O}_{p}^{(1)}\right)$ [6]. The latter correspond to the term $\left(\vec{\partial}^{2}-M_{W} \Delta\right)^{2} /\left(8 M_{W}^{3}\right)$ in (2.2). A comparison of the EFT Born approximations with the full result computed with Whizard [8] shows a good convergence of the series [4]. However partial inclusion of $\mathrm{N}^{3 / 2} \mathrm{LO}$ corrections is necessary to obtain an agreement of $\sim 0.1 \%$ at $170 \mathrm{GeV}$ and $\sim 10 \%$ at $155 \mathrm{GeV}[4]$. 


\section{Radiative corrections}

A complete NLO prediction must include radiative corrections to the Born result. These are electroweak and QCD corrections to the matching coefficient of $\mathscr{O}_{p}^{(0)}$ and loop contributions to the EFT matrix elements. At NLO the flavor-specific final state is selected by multiplying the total cross section with NLO branching ratios. The $O(\alpha)$ correction to the matching coefficient of (3.1) is obtained from the one-loop amplitude of $e^{-} e^{+} \rightarrow W^{-} W^{+}$. Many of the 180 one-loop diagrams do not contribute due to threshold kinematics and the result reads [4]:

$$
C_{p}^{(1)}=\frac{\alpha}{2 \pi}\left[\left(-\frac{1}{\varepsilon^{2}}-\frac{3}{2 \varepsilon}\right)\left(-\frac{4 M_{W}^{2}}{\mu^{2}}\right)^{-\varepsilon}+c_{p}^{(1, \text { fin })}\right]
$$

The one-loop corrections to the matrix elements arise from exchange of potential $\left(\left(q_{0},|\vec{q}|\right) \sim\right.$ $\left.M_{W}(\delta, \sqrt{\delta})\right)$ and soft $\left(\left(q_{0},|\vec{q}|\right) \sim M_{W}(\delta, \delta)\right)$ photons. Loops containing $n$ potential photons are enhanced by inverse powers of $v, \Delta \mathscr{A} \sim \mathscr{A}^{(0)} \alpha^{n} v^{-n} \sim \mathscr{A}^{(0)} \alpha^{n / 2}$, so that the first and second Coulomb corrections must be included in a NLO calculation. Near threshold they amount respectively to $\sim 5 \%$ and $\sim 0.2 \%$ of $\sigma^{(0)}[4]$.

Two-loop diagrams with soft photons connecting different hard subprocesses of (3.1) give the so-called non-factorizable corrections. As a consequence of the residual gauge-invariance of $\mathscr{L}_{\text {eff }}$, and in agreement with previous results [9], only the initial-initial state interferences survive:

$$
{ }_{e}^{e}
$$

with $\eta_{-}=r_{0}-\frac{|\vec{r}|^{2}}{2 M_{W}}+i \frac{\Gamma_{W}^{(0)}}{2}$ and $\eta_{+}=E-r_{0}-\frac{|\vec{r}|^{2}}{2 M_{W}}+i \frac{\Gamma_{W}^{(0)}}{2}$.

\section{Results and remaining theoretical uncertainties}

Because of the approximation $m_{e}=0$, the sum of the corrections calculated in Section 4 is not infrared safe, containing uncanceled $\varepsilon$-poles. The result should be convoluted with $\overline{\mathrm{MS}}$ electron distribution functions after minimal subtraction of the pole. Since the distributions available in the literature are computed in a different scheme, which assumes $m_{e}$ as infrared regulator, it is more convenient to convert our result from $\overline{\mathrm{MS}}$ to this scheme. This is done by adding contributions from the hard-collinear $\left(k^{2} \sim m_{e}^{2}\right)$ and soft-collinear $\left(k^{2} \sim m_{e}^{2} \frac{\Gamma_{W}}{M_{W}}\right)$ regions. These cancel the $\varepsilon$-poles, but introduce large logs of $2 M_{W} / m_{e}$ [4]. The large logs are resummed by convoluting the NLO cross section with the structure functions $\Gamma_{e e}^{\mathrm{LL}}$ used in [2] after subtracting the double counting terms [4]. Since only leading logs are resummed in $\Gamma_{e e}^{\mathrm{LL}}$, one can equivalently choose to convolute only the Born cross section with the structure functions, as done for example in [3], the difference being formally NLL. Fig. 1 shows the percentual correction to the Born result due to initial-state radiation alone (solid black), full NLO corrections with ISR improvement of the Born cross-section only (dot-dashed red), and complete NLO corrections with full ISR improvement (dashed blue). The contribution of genuine electroweak and QCD corrections amounts to $\sim 8 \%$ at threshold. It must also be noted that the difference between the two implementations of ISR is numerically important, 
reaching $\sim 2 \%$ at threshold. A comparison of the EFT approximation with [3] reveals a discrepancy which is never larger than $\sim 0.6 \%$ in the range $161 \mathrm{GeV}<\sqrt{s}<170 \mathrm{GeV}$. More precisely we have for the full calculation $\sigma_{4 \mathrm{f}}(161 \mathrm{GeV})=118.12(8) \mathrm{fb}, \sigma_{4 \mathrm{f}}(170 \mathrm{GeV})=401.8(2) \mathrm{fb}$ [3], while in the EFT one obtains $\sigma_{\mathrm{EFT}}(161 \mathrm{GeV})=117.38(4) \mathrm{fb}, \sigma_{\mathrm{EFT}}(170 \mathrm{GeV})=399.9(2) \mathrm{fb}$ [4].

The dominant remaining theoretical uncertainty comes from an incomplete NLL treatment of ISR. This translates into an uncertainty on the $W$ mass of $\sim$ $31 \mathrm{MeV}$ [4]. Further uncertainties come from $\mathrm{N}^{3 / 2} \mathrm{LO}$ corrections in the EFT. The missing $O(\alpha)$ corrections to the four-electron operator (3.3), which are included in [3], contributes an estimated uncertainty of $\sim 8 \mathrm{MeV}$ [4], while interference of potential and soft photon exchange accounts for additional $\sim 5 \mathrm{MeV}[4]$. This means that with a NLL treatment of initial-state radiation, which seems realistically achievable in the near

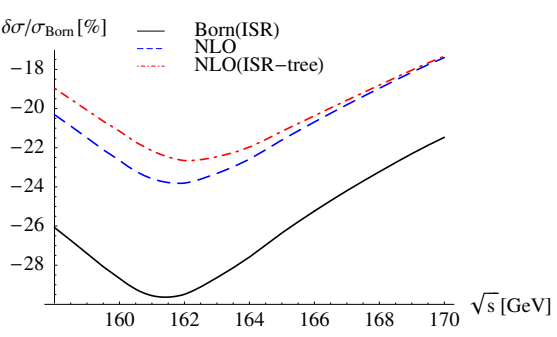

Figure 1: Size of the relative NLO corrections for different implementations of ISR future, and further inputs from [3] the total theoretical error on $M_{W}$ could be reduced to the level required for phenomenological applications at linear colliders.

\section{Acknowledgments}

I thank M. Beneke, C. Schwinn, A. Signer and G. Zanderighi for the collaboration on [4] and for comments on the manuscript.

\section{References}

[1] G. Wilson, in 2nd ECFA/DESY Study, pp. 1498-1505, Desy LC note LC-PHSM-2001-009.

[2] A. Denner, S. Dittmaier, M. Roth and D. Wackeroth, Nucl. Phys. B587, 67 (2000), [hep-ph/0006307]; Phys. Lett. B475, 127 (2000), [hep-ph/9912261]; W. Beenakker, F. A. Berends and A. P. Chapovsky, Nucl. Phys. B548, 3 (1999), [hep-ph/9811481].

[3] A. Denner, S. Dittmaier, M. Roth and L. H. Wieders, Nucl. Phys. B724, 247 (2005), [hep-ph/0505042]; Phys. Lett. B612, 223 (2005), [hep-ph/0502063].

[4] M. Beneke, P. Falgari, C. Schwinn, A. Signer, G. Zanderighi, Nucl. Phys. B792, 89 (2008) [arXiv:0707.0773 [hep-ph]]; C. Schwinn, [arXiv:0708.0730 [hep-ph]].

[5] A. P. Chapovsky, V. A. Khoze, A. Signer and W. J. Stirling, Nucl. Phys. B621, 257 (2002), [hep-ph/0108190].

[6] M. Beneke, N. Kauer, A. Signer and G. Zanderighi, Nucl. Phys. Proc. Suppl. 152, 162 (2006), [hep-ph/0411008].

[7] M. Beneke, A. P. Chapovsky, A. Signer and G. Zanderighi, Nucl. Phys. B686, 205 (2004), [hep-ph/0401002].

[8] W. Kilian, in 2nd ECFA/DESY Study, pp. 1924-1980, DESY LC-Note LC-TOOL-2001-039.

[9] V. S. Fadin, V. A. Khoze and A. D. Martin, Phys. Rev. D49, 2247 (1994); K. Melnikov and O. I. Yakovlev, Phys. Lett. B324, 217 (1994), [hep-ph/9302311]. 